\title{
Propuesta de una guía para la evaluación de la exactitud posicional de datos espaciales
}

\author{
Proposal for a guide for the positional accuracy \\ assessment of spatial data
}

\author{
Francisco Javier Ariza-López* \\ José Luis García-Balboa` \\ Joselyn Robledo Ceballos` \\ José Rodríguez-Avi ${ }^{\bullet}$ \\ Rosario Casanova"
}

Recibido 20 de marzo de 2019; aceptado 13 de junio de 2019

\section{Resumen}

Los participantes en el proyecto de asistencia técnica del IPGH (PAT2018) (Agenda del IPGH 2010-2020) "Propuesta de adopción de metodologías y procedimientos para la evaluación de la calidad de la información geográfica para los Estados Miembros del IPGH", decidieron abordar la realización de una propuesta de guía genérica para la evaluación de la exactitud posicional de datos espaciales. En la elaboración de la propuesta de guía se han aplicado el ciclo de Deming y un método específico de desarrollo de guías basadas en evidencias. Se ha asumido su alineación con las

* Universidad de Jaén, España, correo electrónico: fjariza@ujaen.es. ORCID:http://orcid.org/0000-0002-5204-3630.

- Universidad de Jaén, España, correo electrónico: jlbalboa@ujaen.es. ORCID: http://orcid.org/0000-0002-3109-5888.

- Servicio Aerofotogramétrico de la Fuerza Aérea, Chile, correo electrónico: joselyn.robledo@gmail.com. ORCID: https://orcid.org/0000-0001-6869-3665

- Universidad de Jaén, España, correo electrónico: jravi@ujaen.es. ORCID: https://orcid.org/0000-0002-1673-9876.

- Universidad de la República, Uruguay, correo electrónico: casanova@fing.edu.uy. ORCID: https://orcid.org/0000-0002-1873-207X. 
normas internacionales de la familia ISO 19100 y con los métodos de evaluación de la exactitud posicional más extendidos en Hispanoamérica. Igualmente, se presentan los distintos alcances asumidos para su elaboración, tanto en lo que se refiere a contenidos como a la perspectiva genérica desarrollada.

Palabras clave: exactitud posicional, evaluación de la calidad, guía de evaluación.

\begin{abstract}
The participants in the Technical Assistance Project (TAP2018) of the Pan American Institute for Geography and History entitled "Proposal for the adoption of methodologies and procedures for the evaluation of the quality of the geographic information for the Member States of the PAIGH", have decided to address the realization of a generic guide proposal for the evaluation of spatial data positional accuracy. In the elaboration of the proposal, the Deming cycle and a specific method have been applied to develop evidence-based guides. The assumed lineaments are, the alignment with the international standards of the ISO 19100 family and with the most widespread positional accuracy assessment methods in Latin America stand out. Likewise, the different assumed scopes are presented, both in terms of content and the generic perspective developed. In relation to the contents, the propositive part of the guide, which includes: a general method for the evaluation of positional accuracy, aspects related to execution, aspects related to statistics and meta-quality and a proposal for evaluation report; is the one that receives more dedication in this presentation.

Key words: positional accuracy, quality evaluation, evaluation guide.
\end{abstract}

\title{
Introducción
}

Desde siempre, la exactitud posicional ha sido considerada como un aspecto definitorio y primordial de la calidad de todo producto cartográfico (Ariza-López, 2002), debido a que afecta a factores como la geometría, la topología y la calidad temática, y que está directamente relacionada con la interoperabilidad del dato espacial.

Dada la masificación del uso de la información geoespacial y las necesidades de interoperabilidad que exigen las diferentes aplicaciones geomáticas e Infraestructuras de Datos Espaciales (IDE), es fundamental asegurar la calidad de la información, pues es la única forma de garantizar soluciones fiables al momento de tomar decisiones.

La calidad de los productos de datos espaciales se ha de definir en sus especificaciones (p.ej. utilizando ISO 19131 - ISO, 2007). Los procesos de producción deben estar diseñados y gestionados adecuadamente para que la calidad diseñada sea asegurada en la producción y, así, alcanzada en el producto. Para verificar que lo anterior se consigue, se aplican evaluaciones de la calidad, que deben basarse en métodos 
estandarizados y bien definidos. Los resultados de las evaluaciones deben ser utilizados por los productores para conocer, manejar y mejorar sus procesos de producción. Estos resultados también deben ser publicados en metadatos para que los usuarios tengan conocimiento de la calidad real de los productos que desean utilizar.

Las fuentes de error en las diferentes etapas del proceso productivo son múltiples, variadas y dependientes del propio proceso (p.ej. captura del vuelo, proceso geodésico, proceso de orientación, restitución, captura y estandarización de bases de datos, etc.). Dado este complejo escenario es imposible garantizar la perfección del producto, en nuestro caso: la ausencia de errores posicionales. Lo importante es controlar que el nivel de exactitud existente (sesgos e incertidumbres) esté acotado al final del proceso productivo, y que se cumpla con los estándares internacionales, sus parámetros y tolerancias para cada escala (resolución).

Dentro de este marco, los métodos de evaluación de la exactitud posicional (MEEP) son procesos estandarizados que permiten, o bien estimar la calidad, o controlarla. La estimación consiste en determinar un valor fiable de la propiedad de interés en el producto de datos, mientras que el control de calidad consiste en tomar una decisión de si la propiedad de interés en ese producto de datos alcanza, o no, un nivel determinado.

Existe un trabajo preliminar que permitió obtener un diagnóstico sobre los MEEP en los Países Miembros del Instituto Panamericano de Geografía e Historia (IPGH) a través del proyecto "Diagnóstico de la situación actual sobre las metodologías y procedimientos empleados para la evaluación de la calidad de la Información Geográfica", financiado en la convocatoria de proyectos de asistencia técnica del año 2015. Este trabajo está publicado en la Revista Cartográfica número 94 correspondiente al año 2017 (Ariza-López et al., 2017). En este trabajo se identificaron los MEEP que se están aplicando (Tabla 1), se analizó la situación actual y se valoró el grado de madurez en este aspecto. Con respecto a la situación y a los MEEP utilizados se indica: "La mayoría de los casos analizados tienen como base de la evaluación posicional métodos procedentes de los Estados Unidos. Estos métodos son el NMAS y el NSSDA. La norma del IPGH (1978) también se menciona en algunos países. De modo general, se aprecia un claro interés por mantenerse actualizados y mejorar los procesos de evaluación de la exactitud posicional, lo que lleva a adoptar los cambios que van surgiendo fuera de sus fronteras". Y con respecto a la aplicación de estos MEEP se concluye: “...el principal problema que se detecta es el que se ha denominado "informalidad", es decir, la situación en la que no se dispone de una norma nacional publicada o una referencia explícita a la adopción de un método concreto (p.ej. IPGH, NMAS, NSSDA, etc.)". Además, esta informalidad, no sólo significa que no exista una adopción explícita de un MEEP ya existente o una norma o estándar propio, sino que, por lo general, también faltan documentos detallados que restrinjan la variabilidad de las múltiples interpretaciones y opciones que se pueden desarrollar 
a la hora de aplicar los MEEP existentes. Por otra parte, los autores no conocemos la existencia de ninguna guía que tenga un contenido similar a la desarrollada. El manual sobre la aplicación del NSSDA (MPLMIC, 1999) presenta un carácter aplicado pero muy limitado, y otros documentos centrados en esta materia (p.ej. Poggioli, 2010, o el capítulo 3 de Congalton y Green, 2009) no realizan un tratamiento con suficiente detalle y con una orientación de guía aplicable.

Tabla 1

Uso de estándares identificados en Hispanoamérica

\begin{tabular}{|c|c|c|}
\hline País & Norma & Referencia \\
\hline $\begin{array}{l}\text { Argentina } \\
\text { (Provincia de } \\
\text { Santa Fe) }\end{array}$ & Sí & $\begin{array}{l}\text { UNL\&SCIT (2004). Norma cartográfica de la Provincia } \\
\text { de Santa Fe }\end{array}$ \\
\hline Brasil & Sí & $\begin{array}{l}\text { ET-CQDG (2016): Norma da especificação técnica para } \\
\text { controle de qualidade de dados geoespaciais (ET- } \\
\text { CQDG). } \\
\text { Decreto No } 89.817 \text {, DE } 20 \text { DE JUNHO DE } 1984 . \\
\text { Estabelece as Instruções Reguladoras das Normas } \\
\text { Técnicas da Cartografia Nacional }\end{array}$ \\
\hline Chile & No & Informantes. Se aplican: NSSDA, ASPRS, EMAS \\
\hline Colombia & Sí & $\begin{array}{l}\text { NTC } 5205 \text { - Precisión de datos espaciales } \\
\text { ASPRS (2015). ASPRS Positional Accuracy Standards } \\
\text { for Digital Geospatial Data } \\
\text { IPGH (1978). Especificaciones para mapas topográficos. } \\
\text { Panamá: Instituto Panamericano de Geografía e Historia } \\
\text { - IPGH, Panamá, } 1978\end{array}$ \\
\hline Ecuador & No & Informantes. Se aplica NSSDA \\
\hline España & Sí & $\begin{array}{l}\text { UNE 148002:2016. Metodología de evaluación de la } \\
\text { exactitud posicional de la información geográfica }\end{array}$ \\
\hline México & Sí & $\begin{array}{l}\text { INEGI. Norma técnica sobre estándares de exactitud } \\
\text { posicional } \\
\text { INEGI. Compendio de criterios y especificaciones } \\
\text { técnicas para la generación de datos e informaciones de } \\
\text { carácter fundamental. } 07 \text { geodesia- } 07.2 \text { Estándares de } \\
\text { Exactitud Posicional }\end{array}$ \\
\hline Perú & No & $\begin{array}{l}\text { Instituto Geográfico Nacional normas técnicas " } \\
\text { especificaciones técnicas para la producción de mapas } \\
\text { topográficos a escala de 1:100 000" }\end{array}$ \\
\hline Puerto Rico & Sí & $\begin{array}{l}\text { Estándar TIG-005-004: Exactitud posicional de los } \\
\text { datos. Oficina de Gerencia y Presupuesto }\end{array}$ \\
\hline Uruguay & No & Informantes \\
\hline $\begin{array}{l}\text { Estados } \\
\text { Unidos }\end{array}$ & Sí & $\begin{array}{l}\text { NMAS (USBB, 1947), NSSDA (FGDC, 1998), } \\
\text { ASPRS (2015) }\end{array}$ \\
\hline Venezuela & No & $\begin{array}{l}\text { IGVSB (2012). Especificaciones técnicas para la } \\
\text { revisión de cartografía básica a escala 1:1 } 000 . \\
\text { Instituto Geográfico de Venezuela Simón Bolívar }\end{array}$ \\
\hline
\end{tabular}

Fuente: Ariza-López et al. (2017). 
Finalmente, con base en el análisis realizado en el mencionado trabajo se cita como idea principal a desarrollar la "Visión de Aplicación del Marco Normativo de las Américas" (NN.UU., 2013), el cual indica: "Es preciso definir y adoptar un marco normativo compatible en la región, que establezca las convenciones comunes y acuerdos técnicos necesarios para alcanzar mayor eficiencia en la respuesta a las demandas de información geoespacial, donde los datos se generen y mantengan el común denominador de: compatibilidad, comparabilidad, confiabilidad, consistencia y completitud, siendo éste la base para el establecimiento de un esquema interoperable de colaboración, que contribuya al desarrollo de la Infraestructura de Datos Geoespaciales de las Américas (IDEA)".

Dados los antecedentes expuestos, dentro del proyecto de asistencia técnica del IPGH (PAT2018) (Agenda del IPGH 2010-2020) "Propuesta de adopción de metodologías y procedimientos para la evaluación de la calidad de la información geográfica para los Estados Miembros del IPGH” (https:/coello.ujaen.es/ investigacion/web giic/SubWeb_IPGH2018/index.htm), en el que participaban técnicos de Argentina, Brasil, Chile, Ecuador, España, México y Uruguay, se consideró pertinente la creación de una guía genérica teórico-práctica para la evaluación de la exactitud posicional que pudiera ayudar a los técnicos de los organismos públicos cartográficos, y demás agentes del sector (profesionales, docentes, alumnos, etc.), a mejorar la calidad de los procesos de evaluación de la exactitud posicional.

El objetivo de este trabajo es presentar el método seguido para desarrollar esta guía genérica, así como sus contenidos más sustanciales. El documento se organiza de la siguiente manera: la segunda sección presenta el método aplicado, posteriormente en la tercera se indican los lineamientos y los alcances adoptados; el apartado cuarto muestra una visión general de los contenidos entrando en detalle en aquellos de carácter propositivo que son el propio método general para la evaluación de la exactitud posicional y el informe de evaluación; se finaliza con unas conclusiones.

\section{Método para el desarrollo de la guía}

Una guía ha de ser un documento que sirva de ayuda real al caso propuesto, es decir, que ofrezca recomendaciones de valor para los usuarios, en nuestro caso para la evaluación de la exactitud posicional. Entendemos por recomendación lo que se induce a los usuarios de la guía para realizar una actividad o método de una manera determinada. Por ello, en la creación de una guía se ha de adoptar un método que sea eficaz. Existen numerosas guías para muy diversos temas (p.ej. muestreos estadísticos (EPA, 2002), atlas urbano europeo (EU, 2012), levantamientos topográficos (MSHA, 2001), etc.), pero no es así en lo relativo a cómo desarrollar el propio proceso de elaborar una guía. Basado en el ámbito sanitario (MinSalud, 2014), ArizaLópez (2017) propone una metaguía, es decir, una guía de guías donde se presenta un método genérico que, parcialmente, es el que se ha aplicado en este trabajo para 
realizar la propuesta que se presentará más adelante. Un aspecto relevante de este método es que toda propuesta que se realice en la guía (p.ej. tamaño de muestra, método de observación, etc.), se ha de basar en evidencias, es decir, en cualquier parte de una referencia (p.ej. artículo científico, manual, norma, etc.) que, basada en ensayos, demostraciones o cualquier otro tipo de prueba científica o experiencia, puede sustentar de manera objetiva esa recomendación.

El proceso base de desarrollo de guías es el ciclo PHCA (Planificar, Hacer, Chequear y Actuar) de Deming (Ariza-López, 2002), se aplica de manera global y también de manera iterada dentro de cada una de las siguientes etapas:

- Planificar. En esta fase se incluyen todas las actividades orientadas a definir el alcance del documento a desarrollar, establecer el equipo de trabajo, etc., con vistas a asegurar el éxito del trabajo. Incluye los siguientes pasos:

1. Priorización y focalización en una guía basada en la evidencia. Esta decisión se toma en el seno de una organización competente tomando en consideración criterios de oportunidad, necesidad, etc.

2. Constitución del grupo de trabajo de la guía. Se deben buscar y proponer técnicos competentes en todas las materias implicadas en el desarrollo de la guía, repartiendo responsabilidades según el tamaño del grupo y el funcionamiento que se haya determinado.

3. Identificación y análisis de conflictos de intereses. Este paso es importante cuando se desea realizar una recomendación neutra, sin vínculo con casas comerciales o tecnologías concretas.

4. Definición del alcance y los objetivos de la guía. Se debe establecer la trascendencia y los objetivos iniciales de la guía, así como algún lineamiento básico.

5. Formulación de los casos de uso y requisitos funcionales.

6. Socialización de alcances y objetivos, casos de uso y requisitos.

En el caso de la guía para la evaluación de la exactitud posicional de datos espaciales, la selección y priorización de esta temática se tomó por consenso por parte de los miembros Proyecto. En relación al grupo de desarrollo, dentro del equipo del Proyecto se constituyó un equipo corto formado por la ingeniera Joselyn Robledo Ceballos y el doctor Francisco Javier Ariza López, cuya misión era avanzar en los contenidos de la guía y someterlos al resto del equipo del Proyecto. No se consideró la existencia de conflicto de intereses. Como objetivo se adoptó redactar una guía aplicada que permita desarrollar evaluaciones de la exactitud posicional absoluta de una manera correcta y fiable y que, además, ofreciera suficientes apoyos teóricos y prácticos. Como lineamiento básico se adoptó el marco establecido por las normas de la familia ISO 19100 del Comité Técnico 211 aclarando, en la medida de lo posible, aquellos aspectos de este proceso que 
suelen causar mayor duda. Todas estas decisiones fueron tomadas y compartidas dentro de los miembros del proyecto.

- Hacer (desarrollo). Son todas las actividades orientadas a la elaboración del material de la guía, como documento. De manera genérica se incluyen revisiones de documentación, realización de experimentos o pilotos, redacción del documento, etc., todas ellas dentro del alcance establecido. La Figura 1 presenta un esquema del proceso lógico orientado a generar recomendaciones y que abarca desde el paso 7 al 10, los cuales se describen a continuación. Finalmente, con el conjunto de evidencias se redacta la guía (paso 11). En mayor detalle los pasos son los siguientes:

7. Búsqueda de antecedentes. Consiste en localizar normas, estándares, documentos técnicos, documentos científicos, etc., que traten los distintos contenidos a desarrollar en la guía (p.ej. tamaño de la muestra de evaluación, distribución espacial de los elementos de evaluación, test de normalidad de los errores, etc.).

8. Evaluación general de la calidad de las referencias. Todas las referencias encontradas no tienen el mismo valor. Las referencias deben ser analizadas con una perspectiva crítica, aceptando aquellas que se consideren fundadas y de buena calidad y rechazando las demás. En esto es importante trabajar con la metacalidad de esas referencias. Algunas veces, dada la limitación de la información disponible, este análisis se limita a la reputación de la fuente.

9. Construcción del conjunto de evidencias. Las evidencias que pasan el filtro de calidad del paso 8 se conforman como un conjunto de evidencias que podrá ser utilizado.

10. Formulación de las recomendaciones. Consiste en la redacción sencilla de los ítems de recomendación que se incluirán en la guía.

11. Redacción de la guía. Es la fase final de compilación ordenada y sistemática de las recomendaciones que se ofrecerán.

Se acaban de describir los pasos de este proceso genérico, pero el desarrollo dentro del Proyecto no ha sido exactamente el arriba indicado pues se ha contado con la participación del equipo de expertos en evaluación de la exactitud posicional de la Universidad de Jaén. Este equipo posee una amplia experiencia investigadora sobre la evaluación de la exactitud posicional y en el desarrollo de estándares y guías, por lo que los pasos 7 al 10 se han podido realizar de una manera más ágil. Un elemento clave de esta propuesta es el manual Fundamentos de evaluación de la calidad de la Información Geográfica (Ariza-López, 2013).

- Chequear (validación). El alcance de esta fase son las actividades de validación externa del documento elaborado en la fase anterior y su aplicación por parte de los usuarios.

12. Revisión por pares (evaluación externa de primer nivel). Antes de su publicación, todo documento se ha de revisar por pares externos e independientes, es 
decir, por terceras personas no pertenecientes al grupo de trabajo, que posean competencia en la materia y que, igualmente, no tengan conflicto de intereses.

- Difusión y revisión pública (evaluación externa de segundo nivel). La difusión es la publicación. Con ella los usuarios tienen constancia de su existencia y conocen sus contenidos. A lo largo de todo el periodo de vigencia la guía se ha de favorecer que los usuarios puedan realimentar el proceso con sus aportaciones (p.ej. comentarios, dudas, experiencias, etc.).

13. En nuestro caso, la revisión por pares de la Guía la realizó un panel reducido de expertos internacionales. En cuanto a la publicación, se le ha asignado el número 557 de la colección de Publicaciones Ocasionales del IPGH y está disponible en http://publicaciones.ipgh.org/publicaciones-ocasionales/Guia Evaluacion_Exactitud_Posicional_Datos_Espaciales.pdf. En la guía se incorpora una dirección de correo electrónico para enviar la retroalimentación por parte de los usuarios.

- Actuar (mejora). El alcance de esta fase son las actividades de revisión del documento en base a las aportaciones recibidas por parte de los usuarios o de los nuevos conocimientos y experiencias disponibles en el campo técnico y científico. 14. Mejora. Son cada uno de los ciclos de revisión que ha de sufrir el documento. En nuestro caso, esta fase todavía no se ha iniciado. Se realizará con mayor o menor rapidez en función del número y tipología de las aportaciones recibidas en el proceso de realimentación.

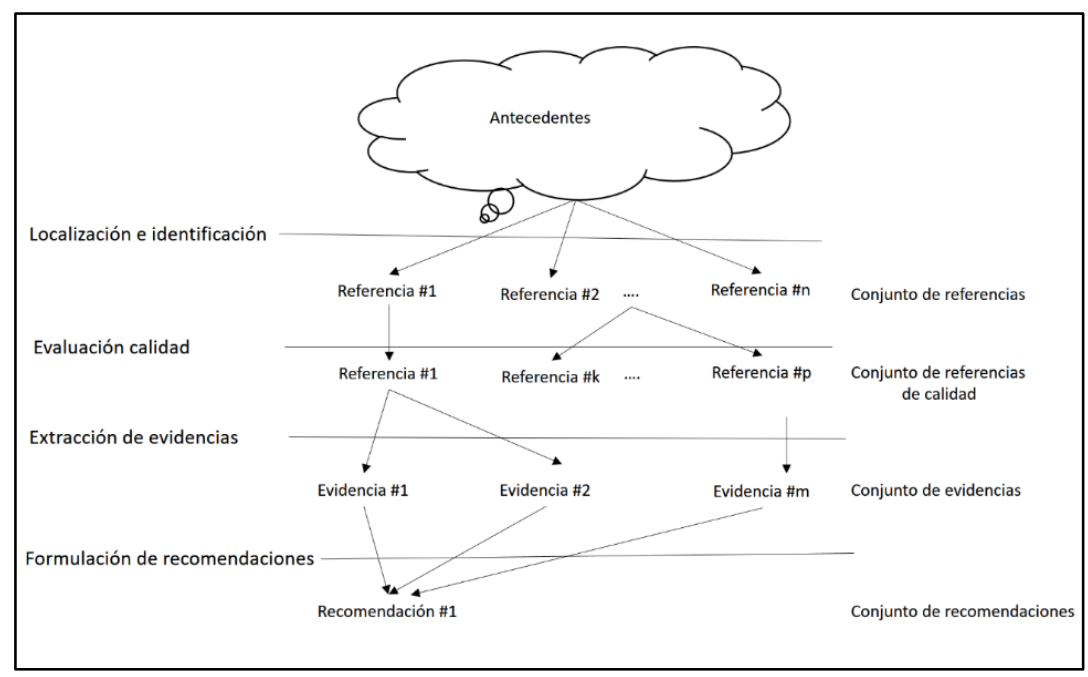

Figura 1. Proceso lógico para derivar recomendaciones basadas en evidencias.

Fuente: Ariza-López (2017). 


\section{Lineamientos y alcance de la propuesta}

Es importante que una guía como la que se propone esté anclada en bases sólidas y aceptadas, tanto científicas como sociales o de aplicación. Por ello, a la hora de considerar su desarrollo se consideraron fundamentales los siguientes lineamientos:

- Lineamiento con las normas internacionales de la familia ISO 19100. Esto supone adoptar el modelo de la Norma Internacional ISO 19157 (ISO 2013) para describir, evaluar e informar sobre la calidad de los datos. Junto a ISO 19157 también se deben considerar otras normas como son:

- ISO 19131 (ISO 2007). Tiene como propósito el establecer directrices para la elaboración de especificaciones de productos de datos. La evaluación de la exactitud posicional deberá ser consistente con lo indicado en las especificaciones del producto con el que se esté trabajando.

- ISO 19115-1 (ISO 2014). Ofrece un modelo de metadatos para datos espaciales, dentro del cual cabe la información relativa a los resultados de la evaluación de la calidad. Estos metadatos pueden ser complementados con el informe de la calidad independiente que se propone en la Norma Internacional ISO 19157.

Junto a lo anterior, en la redacción y presentación de la guía también se ha adoptado el modo y estructura que se utiliza en las normas internacionales.

- Lineamiento con los métodos de evaluación de la exactitud posicional más utilizados en Hispanoamérica. Como resultado del Proyecto "Diagnóstico de la situación actual sobre las metodologías y procedimientos empleados para la evaluación de la calidad de la Información Geográfica" (https://coello.ujaen.es/investigacion/web_giic/SubWeb_IPGH2016/), financiado en la convocatoria de Proyectos de Asistencia Técnica del año 2015 se disponía de una publicación (Ariza-López y col., 2017) que indicaba los métodos base de evaluación de la exactitud posicional más extendidos en los Países Miembros del Instituto Panamericano de Geografía e Historia. De esta forma, se consideró adecuado abarcar los métodos NMAS (National Map Accuracy Standard) (USBB, 1947), EMAS (Engineering Map Accuracy Standard) (ASCE, 1983) y NSSDA (National Standard for Spatial Data Accuracy) (FGDC, 1998). Con ellos, además, se ofrecen tres perspectivas complementarias desde el punto de vista estadístico, lo cual ofrece opciones múltiples a los usuarios y enriquece la guía.

En relación al alcance de sus contenidos, se consideraron las siguientes premisas generales: 
- Parte conceptual: ha de ser abarcadora. La guía debía presentar un marco conceptual esclarecedor sobre los múltiples aspectos que suelen ser problemáticos a la hora de realizar evaluaciones de la exactitud posicional.

- Parte aplicada: ha de ser de ámbito general. La guía debía ofrecer directrices generales para los procesos de diseño de la evaluación, para la determinación de los datos de referencia (p.ej. por trabajos de campo), y para los procesos de cálculo e informe de los resultados. Cada organización puede elaborar su propia guía a partir de esta propuesta general.

En relación al alcance de aplicabilidad, se consideró lo siguiente:

- Elementos de evaluación: han de ser de geometría puntual. La guía se debía limitar a la tradicional evaluación de la exactitud posicional por medio de elementos puntuales por ser la opción operativa actualmente más extendida.

Finalmente, dentro de su alcance se consideró oportuno no ofrecer deliberadamente exigencias de conformidad dado el mero carácter de orientación que se ha pretendido.

\section{Contenidos de la guía}

Los contenidos que se han desarrollado dentro de la guía para la evaluación de la exactitud posicional de datos espaciales son los que se presenta en la

Tabla 2 donde, además, se muestra el carácter de ese contenido. Existen contenidos de introducción al documento, de especificación del propio documento y de apoyo al documento. Las partes más relevantes son, por un lado, los contenidos que establecen el marco conceptual, donde se explica el modelo ISO 19100, algunos aspectos concretos de la exactitud posicional, las diferencias entre estimación versus control y entre métodos de base paramétrica y no paramétrica y, finalmente, los tres métodos base de evaluación de la exactitud posicional más utilizados en Hispanoamérica (NMAS, EMAS y NSSDA) y, por otro, los contenidos de carácter propositivo (general o aplicado), pues estos últimos son los que tienen una vocación de guía. También existen dos contenidos de apoyo a la aplicación de la guía: un conjunto de preguntas frecuentes y un ejemplo de aplicación detallado centrado en el cálculo y en el informe.

En los dos siguientes subapartados se presentarán los contenidos de tipo propositivo general: primeramente, el método general para la evaluación de la exactitud posicional y, posteriormente, el informe de evaluación propuesto.

\section{Método general para la evaluación de exactitud posicional}

Se va a considerar que la evaluación de la exactitud posicional de un producto de datos espaciales se realiza comparando las posiciones de una selección (muestra) 
Tabla 2

Guía para la evaluación de la exactitud posicional de datos espaciales: contenidos y carácter

\begin{tabular}{|c|c|}
\hline Contenido & Carácter \\
\hline Presentación & Introductorio \\
\hline Introducción & \\
\hline $\begin{array}{l}\text { Objetivo } \\
\text { Alcance } \\
\text { Conformidad }\end{array}$ & Especificativo \\
\hline Referencias normativas & Apoyo al documento \\
\hline Términos y definiciones & \\
\hline Abreviaturas & \\
\hline $\begin{array}{l}\text { Calidad de datos espaciales basada en normas ISO del } \\
\text { TC/211 } \\
\text { La norma ISO } 19157 \text { aplicada a la evaluación de } \\
\text { exactitud posicional } \\
\text { Exactitud y error posicional } \\
\text { Estándares de exactitud posicional } \\
\text { NMAS } \\
\text { EMAS } \\
\text { NSSDA }\end{array}$ & Marco conceptual \\
\hline $\begin{array}{l}\text { Método general para la evaluación de exactitud } \\
\text { posicional }\end{array}$ & Propositivo general \\
\hline $\begin{array}{l}\text { Aspectos relativos a la ejecución } \\
\text { Datos de referencia para la evaluación } \\
\text { Exactitud de los trabajos de evaluación } \\
\text { Cantidad y distribución de los elementos de } \\
\text { evaluación } \\
\text { Identificación y observación de los elementos de } \\
\text { evaluación en campo } \\
\text { Identificación y observación de alturas en campo } \\
\text { Identificación y observación de elementos en } \\
\text { imágenes } \\
\text { Aspectos estadísticos } \\
\text { Aspectos de metacalidad de la evaluación de la exactitud } \\
\text { posicional }\end{array}$ & Propositivo aplicado \\
\hline Informe de la evaluación & Propositivo general \\
\hline Referencias & Apoyo al documento \\
\hline $\begin{array}{l}\text { Preguntas y respuestas frecuentes } \\
\text { Anexo 1. Ejemplos de cálculo }\end{array}$ & Apoyo a la aplicación \\
\hline
\end{tabular}


de objetos de tipo puntual del producto, o conjunto de datos a evaluar - $\mathrm{CDE}-$, frente a las posiciones de los objetos puntuales homólogos en una referencia más exacta que el producto, o conjunto de datos de referencia - CDR. De esta forma el proceso es el siguiente:

- Definición del proceso de evaluación. Si existen unas especificaciones de producto adecuadas, los aspectos clave de la evaluación se extraerán de ellas. En otro caso, deberán ser definidos de manera previa a la evaluación. Estos aspectos son:

- El elemento de calidad. Podrá ser cualquiera de los establecidos por ISO 19157 (p.ej. exactitud posicional absoluta o externa).

- El ámbito de la evaluación de la calidad. Se establecerán los aspectos temáticos, espaciales, temporales, etc., que delimiten de manera adecuada el conjunto de objetos que forman parte de la evaluación (p.ej. capa temática de construcciones).

- Unidad de calidad de datos. Es la conjunción de los dos anteriores.

- Nivel de conformidad. En el caso de un control de calidad, deberá existir un nivel o niveles de conformidad que se utilice para tomar la decisión de aceptación/rechazo del producto.

- Método de evaluación. Debe ser la especificación del procedimiento de evaluación de forma integral. No basta con indicar la aplicación de un estándar de evaluación de la exactitud posicional (p.ej. NMAS, EMAS, NSSDA). El método de evaluación al que nos estamos refiriendo debe abarcar todos los aspectos relevantes de la evaluación incluyendo: exactitud necesaria en los trabajos de evaluación, tamaño y generación de la muestra de evaluación, directrices de trabajo en campo (p.ej. técnicas de levantamiento, casos especiales, etc.), procesado estadístico de los datos (p.ej. verificación de hipótesis básicas, tratamiento de valores atípicos, etc.), entre otros. Esta guía es un documento adecuado para orientar en detalle estos contenidos.

- Medida de la calidad. La(s) medida(s) de la calidad a utilizar debe(n) quedar especificada(s) (p.ej. medida ID 45 de ISO 19157).

- Aseguramiento del proceso de evaluación. Una vez se dispone de todos los aspectos que conforman la definición del proceso de evaluación de la exactitud posicional, se deberá proceder a asegurar que realmente se aplicará de manera adecuada. Para ello se consideran diversos aspectos del aseguramiento, todos ellos interrelacionados. Éstos son:

- Aseguramiento del personal. Consiste en disponer de personal cualificado. El personal que vaya a realizar los trabajos de campo (p.ej. observaciones GNSS) y gabinete (p.ej. análisis estadísticos), deberá disponer de la formación y capacitación adecuadas. 
- Aseguramiento del instrumental. Consiste en disponer de equipos físicos (p.ej. receptores GNSS) y de recursos computacionales (p.ej. programas de cálculo) que sean los adecuados al método y exactitudes pretendidas. Todos los equipos y sistemas deben estar calibrados.

- Aseguramiento estadístico. Consiste en disponer de una muestra suficiente (cantidad), representativa (distribución) en la que las hipótesis estadísticas (aleatoriedad, independencia, ausencia de sesgo) y de calidad (mayor exactitud) se cumplan. En relación al tamaño de muestra a utilizar en la evaluación, se deberá tener especial cuidado en distinguir si se trata de una estimación, donde se debe aplicar la teoría de muestreos, o de un control de calidad, donde el tamaño de muestra vendrá condicionado por los riesgos de usuario y productor que se deseen asumir.

- Aseguramiento de los procesos. Todos los procediminentos estarán descritos y serán comprendidos por todo el personal involucrado con el objetivo de conseguir una evaluación fiable y económico. Dentro de este aseguramiento se incluye la planificación de los trabajos de campo (p.ej. rutas, fechas y horas de los trabajos de observación GNSS, etc.), según es usual en los proyectos de ingeniería.

- Trabajo de campo para la evaluación. Se ejecutarán los trabajos de campo consistentes en la realización de las observaciones precisas sobre el terreno de los objetos (puntos) que forman el CDR. Aspectos relevantes de este proceso son:

- Identificación inequívoca de los puntos. No puede existir equivocación en la identificación en la referencia (p.ej. en la realidad o campo) de los puntos que forman el CDR, y además, deberán generarse evidencias de la identificación (p.ej. croquis, fotografías, etc.).

- Observación. El método de levantamiento seleccionado (p.ej. estático rápido) deberá ejecutarse siguiendo un procedimiento estandarizado previamente por la organización, y tal que se asegure la obtención de la exactitud propia de ese método (p.ej. evitando el efecto multicamino e interferencias electromagnéticas).

- Trabajo de gabinete. Se ejecutarán los trabajos de procesado en gabinete consistentes en la obtención de las coordenadas precisas de los objetos (puntos) que forman el CDR. Aspectos relevantes de este proceso son:

- Procesado de observaciones. Las observaciones de campo serán procesadas mediante una herramienta de cálculo validada y siguiendo un procedimiento estandarizado previamente por la organización, y tal que se asegure la obtención de exactitud propia de ese método e instrumental.

- Identificación inequívoca en el producto a evaluar de los puntos homólogos a los CDR, que constituirán el CDE. Deberá asegurarse que no se comente equivocación. 
- Verificación de prerrequisitos cartográficos. Se deberá verificar que las coordenadas del CDR y del CDE corresponden a un mismo sistema geodésico de referencia y proyección cartográfica.

- Verificación de hipótesis estadísticas sobre el error posicional. Se deberá verificar que se cumplen las hipótesis estadísticas que se requieran como base para la aplicación del método estadístico que se utilice para el análisis (p.ej. normalidad, homocedasticidad, atípicos, etc.). Se seguirá un procedimiento estandarizado previamente por la organización para este caso.

- Aplicación de unos de los MEEP estandarizados (p.ej. NMAS, EMAS; NSSDA).

- Análisis e informe. Se examinarán los resultados numéricos, para obtener salidas gráficas (p.ej. mapa de distribución de errores, etc.). Siguiendo un procedimiento estandarizado previamente por la organización para este caso (p.ej. una plantilla de resultados).

Estos aspectos son complementados en la guía de una manera más concreta con las sugerencias que se realizan en los apartados posteriores, dedicados a los aspectos de la ejecución, estadística y metacalidad.

En lo relativo a la ejecución, se incide especialmente a la identificación los puntos en el CDE y el CDR, incluyendo el caso de trabajo con MDE y con imágenes.

En referencia a la estadística se incide en: i) que la muestra de errores debe ser aleatoria, ii) que la normalidad de los errores no siempre se cumplirá y esto es crítico para métodos basados en esta hipótesis, iii) que los valores atípicos deben ser analizados de manera independiente, iv) que la presencia de sesgo relevante debe ser analizada, v) que la homocedasticidad debe ser verificada y vi) que los errores de las distintas componentes, $\mathrm{x}, \mathrm{y}, \mathrm{z}$, deben ser independientes.

En cuanto a la metacalidad, se incide en la importancia de aplicar un método general adecuado y bien asegurado que permita ofrecer elementos de juicio relativos a la metacalidad de los resultados y sus procesos de obtención.

\section{Informe de la evaluación}

Finalmente, un aspecto relevante de toda evaluación es la manera de informar sobre los resultados obtenidos y, en esta línea, se adopta la perspectiva del informe de calidad, independientemente que se menciona en la norma internacional ISO 19157. Para ello en la Guía se propone el esquema que se presenta en la Tabla 3, que se inspira, en parte, en un ejemplo incluido en la norma UNE 148002 (2016). Se trata de un informe bastante exhaustivo y detallado que se propone vincular con aquellos aspectos que cada organización considere oportunos para obtener la conformidad respecto al método de evaluación que diseñe y aplique. A continuación se explica cada uno de los bloques de esta propuesta: 
Tabla 3

\section{Esquema de contenidos de un informe de calidad independiente} para la evaluación de la exactitud posicional

\section{Contenidos}

1) Identificación del producto de datos a $\underline{\text { evaluar }}$

Nombre

ID

Productor

Descripción cualitativa

Propósito

Especificaciones

Exactitud de diseño (teórica)

2) Aspectos generales de la evaluación

Elemento de la calidad

Ámbito

Medidas de la calidad

Niveles de conformidad

Método de evaluación

3) Fuente de mayor exactitud y lista de coordenadas

Fuente de referencia

Dimensión

Exactitud de la referencia

Recubrimiento: poblacional, temático, espacial

Interoperabilidad

Aseguramiento de la aleatoriedad de la muestra

4) Comprobación de las hipótesis, estadísticas de los errores

Lista de errores

Aleatoriedad

Atípicos

Normalidad

Sesgos

Independencia

Homocedasticidad

Interpretación de las comprobaciones
Contenidos (cont.)

5) Resultados

Lista de errores definitiva

(sin atípicos)

Parámetros estadísticos básicos

Diagrama circular de distribución

de errores $\mathrm{X}, \mathrm{Y}$

Diagrama de distribución de errores Z

Histogramas de los errores

Distribución espacial de los errores

Distribución espacial de los atípicos

Lista de coordenadas y errores

Asignación del sesgo

Resultados de las medidas y

conformidad

Resultados de los MEEP

Interpretación de los resultados

6) Metacalidad de los resultados

y procesos

Confianza

Homogeneidad

Representatividad

7) Fecha y firma del

responsable

Fecha

Firma 
- Identificación del producto de datos a evaluar. Reconoce el producto y describe los aspectos más relevantes (p.ej. contenido, propósito, especificaciones), así como las especificaciones propias de la exactitud posicional.

- Aspectos generales de la evaluación. Se establece la unidad de calidad de datos para identificar y comunicar de manera clara los elementos y ámbitos de la calidad que se evalúan. Igualmente se debe identificar la medida, niveles de conformidad (si los hubiera) y el método de evaluación utilizados de tal manera que no quede ambiguo ningún aspecto relevante (tal como se ha indicado en los distintos apartados de esta guía). Para ello, al objeto de no hacer demasiado extenso el informe, se aconseja resumir el método de evaluación e introducir referencias a documentos externos que aporten más detalles (por ejemplo, ejecución de trabajos en campo, en gabinete, etc.).

- Fuente de mayor exactitud (CDR) y lista de coordenadas. Esta parte de informe de evaluación tiene mucha importancia desde la perspectiva de la metacalidad, por ello se incluyen contenidos que han de permitir apreciar la calidad del CDR. Se trata pues de un apartado crítico dentro del informe. Se puede aportar información sobre: cuál es la fuente de referencia, si es planimétrica y/o altimétrica, su exactitud, el recubrimiento poblacional (tamaño de muestra), temático o espacial, si se ha comprobado la interoperabilidad (que el CDE y el CDR se encuentren en el mismo sistema de referencia), cómo se ha generado la muestra, etc.

- Comprobación de las hipótesis estadísticas sobre los errores. Esta parte del informe se centra en mostrar las evidencias relativas a la comprobación de que todas las hipótesis estadísticas, ya sean implícitas o explícitas, y que son requeridas por el/los MEEP aplicado/s, se satisfacen.

- Resultados. Incluye los resultados finales (cumple/no cumple o un valor de medida) que ofrece el método o métodos aplicados. También debe incluir las distribuciones de los errores y demás parámetros en las formas relevantes (espacial, histograma, etc.). Si existía sesgo y ha sido asignado se debe explicar. Finalmente, es conveniente una breve interpretación de todos los resultados de manera conjunta.

- Metacalidad de los resultados y procesos. En esta parte del informe se desarrollarán explicaciones justificativas relativas a los elementos de la metacalidad. Deberán estar basadas en hechos objetivos presentados en los apartados 2, 3, 4, 5 y 6 (Tabla 3).

- Fecha y firma del responsable. Toda evaluación debe tener asignada una fecha y un técnico responsable que ha de aprobarla.

\section{Conclusión}

Se ha presentado la justificación de la necesidad de elevar el nivel de estandarización de los procesos de evaluación de la exactitud posicional y cómo ello ha motivado que en el seno del Proyecto de Asistencia Técnica del IPGH (PAT2018) 
(Agenda del IPGH 2010-2020) "Propuesta de adopción de metodologías y procedimientos para la evaluación de la calidad de la información geográfica para los Estados Miembros del IPGH", se haya abordado la realización de una propuesta de una guía genérica para la evaluación de la exactitud posicional de datos espaciales. La guía desarrollada no pretende ser de aplicación directa, pues cada organización tiene un saber hacer, y unas condiciones propias, que no permiten establecer un proceso común. No obstante, aun siendo genérica, esta propuesta de guía aborda de manera conjunta e interrelacionada temas que suelen generar disparidad de criterios y formas de trabajo, lo que origina variabilidad en los resultados y falta de posibilidad real de comparación. Se propone un método general para la evaluación de exactitud posicional, acompañado de aspectos más prácticos, y un entorno explicativo que permiten entender mejor qué y cómo se deben hacer las cosas en una evaluación de la exactitud posicional. Además, la guía ofrece un esquema, también general, de informe, que puede ayudar a entender mejor los procesos de evaluación de la exactitud tanto a los productores como a los usuarios y donde se han introducido contenidos de metacalidad. Para la elaboración de la guía se ha adoptado un método basado en evidencias, de tal manera que se tiene gran confianza en todo lo propuesto.

Son muchos los aspectos genéricos y detalles que podrían añadirse a la guía desarrollada, pero consideramos que es única en su concepción y que aporta valor al sector. Esperamos que tenga buena acogida y que pronto los usuarios nos remitan sus comentarios para seguir manteniéndola y mejorándola.

\section{In memoriam}

Queda en nuestra memoria y agradecimiento Edison Rojas, nuestro compañero y amigo, que solicitó este proyecto y que nos ha dejado sin ver este fruto, que también se debe a su esfuerzo y liderazgo.

\section{Agradecimientos}

Al Instituto Panamericano de Geografía e Historia, especialmente a las comisiones de Cartografía y Geografía por financiar dentro del programa de Proyectos de Asistencia Técnica 2018 (Agenda del IPGH 2010-2020) el proyecto "Propuesta de adopción de metodologías y procedimientos para la evaluación de la calidad de la información geográfica para los Estados miembros del IPGH".

\section{Bibliografía}

Ariza-López, F.J., Xavier E., Chicaiza E.G., Buenaño X. (2017). "Métodos de evaluación de la calidad posicional en Hispanoamérica: análisis de la situación", en Revista Cartográfica, núm. 94, pp. 65-88. Recuperado de https://revistasipgh.org/index.php/rcar/article/view/342 
Ariza-López, F.J. (2002). “Calidad en la producción cartográfica”. RA-MA, Madrid. (2017). "Documento P1.2. Plan para el desarrollo de guías de implementación. Anexo: Guía para el desarrollo de Guías Prácticas", Proyecto de Modernización de la Administración de Tierras en Colombia, Agencia de Implementación, Bogotá.

— (ed.) (2013). "Fundamentos de evaluación de la calidad de la Información Geográfica”, Universidad de Jaén.

ASCE (1983). "Map Uses, scales and accuracies for engineering and associated purposes", American Society of Civil Engineers, Committee on Cartographic Surveying, Surveying and Mapping Division, New York, USA.

ASPRS (2015). “ASPRS Positional accuracy standards for digital geospatial data", Photogrammetric Engineering \& Remote Sensing, 81(3), 53 pp. Recuperado de http://www.asprs.org/a/society/divisions/pad/Accuracy/Draft_ASPRS_Accuracy Standards_for_Digital_Geospatial_Data_PE\&RS.pdf

Congalton, R.G. and Green, K. (2009). Assessing the Accuracy of Remotely Sensed Data: Principles and Practices, 2nd Edition, Lewis Publishers, Boca Raton.

EPA (2002). "Guidance for Choosing a Sampling Design for Environmental DataCollection (EPA QA/G-5S)", U.S. Environmental Protection Agency.

EU (2012). "Mapping Guide for a European Urban Atlas”. Ref. Ares (2012)1348219 - 15/11/2012, European Commission.

FGDC (1998). "FGDC-STD-007: Geospatial Positioning Accuracy Standards, Part 3. National Standard for Spatial Data Accuracy", Federal Geographic Data Committee, Reston, USA. Recuperado de https://www.fgdc.gov/standards/projects/accuracy/part3/chapter3

IPGH (1978). Instituto Panamericano de Geografía e Historia: Especificaciones para mapas topográficos, Panamá, Instituto Panamericano de Geografía e Historia.

ISO (2014). “19115-1:2014 Geographic information - Metadata -Part 1: Fundamentals", International Ogranization for Standardization.

ISO (2007). ISO 19131:2007 Geographic information - Data product specifications. International Ogranization for Standardization.

ISO (2013). ISO 19157:2013 Geographic information - Data quality. International Ogranization for Standardization.

MinSalud (2014). "Guía Metodológica para la elaboración de Guías de Práctica Clínica con Evaluación Económica en el Sistema General de Seguridad Social en Salud Colombiano", Ministerio de Salud y Protección Social, Santa Fé de Bogotá, Colombia.

MPLMIC (1999). "Positional Accuracy Handbook: Using the National Standard for Spatial Data Accuracy to measure and report geographic data quality", Minnesota 
Planning Land Management Information Center. Recuperado de https://www.mngeo.state.mn.us/pdf/1999/1mic/nssda o.pdf

MSHA (2001). "Survey field procedures manual", The Division of Plats and Surveys, Maryland State Highway Administration.

NN.UU. (2013). "Visión del Marco normativo de las Américas". Naciones Unidas. Comité permanente de datos geoespaciales. Recuperado de https://unstats.un.org/unsd/geoinfo/RCC/docs/rcca10/E\%20Conf 103 15 CPIDEA Marc oNormativo ESP final.pdf

Poggioli D. (2010). "Spatial data quality in SDI environment". LAP Lambert Academic Publishing.

UNE (2016). "UNE 148002:2016 Metodología de evaluación de la exactitud posicional de la información geográfica", UNE, Madrid.

USBB (1947). "United States National Map Accuracy Standards”, U.S. Bureau of the Budget, Washington, USA. 
